Research article

Open Access

\title{
Synthesis and Biological Evaluation of Novel Alkyl-Imidazolyl Carbinols and their Esters: Potent Antimycotics
}

\author{
Jürgen Krauss, Carina Gratzl, Verena Sturm, Christoph MüLler, \\ Verena Staudacher, Christoph Q. SchmidT, Franz BRACHER *
}

Department of Pharmacy - Center for Drug Research, Ludwig-Maximilians University, Butenandtstr. 5-13, 81377 Munich, Germany.

* Corresponding author. E-mail: Franz.Bracher@cup.uni-muenchen.de (F. Bracher)

Sci Pharm. 2013; 81: 641-650

doi:10.3797/scipharm.1304-17

Published: $\quad$ September $2^{\text {nd }} 2013$

Received: $\quad$ April $17^{\text {th }} 2013$

Accepted: $\quad$ August $21^{\text {st }} 2013$

This article is available from: http://dx.doi.org/10.3797/scipharm.1304-17

(c) Krauss et al.; licensee Österreichische Apotheker-Verlagsgesellschaft m. b. H., Vienna, Austria.

This is an Open Access article distributed under the terms of the Creative Commons Attribution License (http://creativecommons.org/licenses/by/3.0/), which permits unrestricted use, distribution, and reproduction in any medium, provided the original work is properly cited.

\begin{abstract}
A novel series of imidazol-5-yl carbinols and their 4-chlorobenzoyl esters has been synthesized by the Grignard reaction and subsequent esterification. These compounds were screened for their antimicrobial activities in an agar diffusion assay. The compounds with $\mathrm{C}_{10}$ to $\mathrm{C}_{12}$-alkyl side chains displayed significant antimycotic activity.
\end{abstract}

\section{Keywords}

Grignard reaction • Ergosterol biosynthesis • Sterol biosynthesis inhibitors • Antimycotics

\section{Introduction}

Ergosterol biosynthesis is an important target for the development of novel antimycotic drugs [1-8]. Three classes of antimycotics, the azoles (e.g. fluconazole, clotrimazole, and miconazole), the allylamines (e.g. naftifine, terbinafine), and the morpholines (amorolfine) are used in the treatment of human mycoses caused by various species of fungi (Fig. 1). Diverse derivatives of these drug classes are also used in agrochemistry for crop protection or in technical chemistry (e.g. as additives in colourings).

These drugs target enzymes in fungal ergosterol biosynthesis. While azoles target C-14 demethylase [7], allylamines inhibit the enzyme squalene epoxidase, and the morpholines inhibit both $\Delta 8,7$-isomerase and $\Delta 14$-reductase. 
Increasing fungal resistance against these antimycotics [2] and their often observed unfavourable pharmacokinetics call for the development of new antimycotics with new targets and better biopharmaceutical properties.

It is well-established that a number of enzymatic transformations in the post-squalene part of ergosterol biosynthesis go through carbocationic intermediates (high-energy intermediates, $\mathrm{HEI}$ ), e.g. those catalyzed by the enzymes sterol $\Delta 8,7$-isomerase, C24-methyltransferase, $\Delta 24,28$-reductase, and $\Delta 14$-reductase [5]. Inhibition of these enzymes can be achieved effectively by imitation of the respective $\mathrm{HEI}$, since mimics of $\mathrm{HEI}$ have a higher affinity for the active site than the substrate sterols have [4]. Synthetic drugs which imitate $\mathrm{HEI}$ have to be cationic in the cellular environment, which is most conveniently achieved by the presence of an aliphatic amino group or heteroaromatic ring (which are protonated to a significant extent at physiological $\mathrm{pH}$ ). The allylamines and the morpholine antifungal amorolfine are the most prominent representatives of this type (Fig. 1). In the class of morpholines and related $N$-alkyl piperidines, the length of the aliphatic or arylaliphatic $N$-substituent significantly determines the potency and selectivity $(\Delta 8,7$-isomerase versus $\Delta 14$-reductase inhibition) of the compounds [9]. On the other hand, the azole-type antifungals (imidazoles like clotrimazole and miconazole, triazoles like fluconazole, as well as numerous related azoles used in agrochemistry [10]) are inhibitors of the enzyme sterol C14 demethylase. This enzyme, a member of the CYP family, catalyzes the oxidative demethylation of lanosterol, and inhibition is based on the complexation of its heme iron by one of the nitrogen atoms of the unprotonated azole ring. Most of the commercial azole antifungals contain a 1-(2-hydroxyethyl)azole subunit (see fluconazole, Fig. 1), but etherification (miconazole) and esterification [11] of the hydroxyl group is tolerated well. The azoles further inhibit another CYP-enzyme in ergosterol biosynthesis, namely $\Delta 22$-desaturase [12]. This enzyme, catalyzing the introduction of an olefinic double bond in the sterol side chain, has been poorly characterized until now, and its selective inhibitors are not yet known. Previously synthesized sterol derivatives containing imidazole groups in the side chain have not inhibited this enzyme, but the sterol C24-methyltransferase has, due to an imitation of a cationic HEI by the protonated imidazole [13].

In the investigations described here, we intended to prepare hybrids of the abovementioned types of established antifungals, aimed at the development of new inhibitors of ergosterol biosynthesis, probably with dual action.

As the central functionality, we selected the imidazole ring ( $p K_{a}$ value about 7 ), which should exist in protonated and unprotonated forms in about equal amounts in cells. In its neutral form it can inhibit the relevant CYP enzymes, whereas the protonated form might imitate carbocationic $\mathrm{HEI}$ in sterol biosynthesis [13]. In contrast to established azole antifungals [10], in which the hydroxyalkyl residue is attached to N-1, our target compounds contain side chains (derived from the alkyl chains of morpholine antifungals [9]) at C-5 of the imidazole ring. 
<smiles>OC(Cn1cncn1)(Cn1cncn1)c1ccc(F)cc1F</smiles>

fluconazole<smiles>CN(C/C=C/c1ccccc1)Cc1cccc2ccccc12</smiles>

naftifine<smiles>Clc1ccccc1C(c1ccccc1)(c1ccccc1)n1ccnc1</smiles>

clotrimazole<smiles>Clc1ccc(COC(Cn2ccnc2)c2ccc(Cl)cc2Cl)c(Cl)c1</smiles>

miconazole<smiles>CN(C/C=C/C#CC(C)(C)C)Cc1cccc2ccccc12</smiles>

terbinafine<smiles>CCC(C)(C)c1ccc(CC(C)CN2C[C@H](C)O[C@H](C)C2)cc1</smiles>

amorolfine

Fig. 1. Antimycotic drugs

\section{Results and Discussion}

\section{Chemistry}

In order to achieve a hydroxyalkylimidazole structural element, we selected a Grignard reaction as the crucial step. Commercially available 1-methyl-1H-imidazole-5-carbaldehyde (1) was reacted with a series of alkyl and arylalkyl magnesium bromides to give the racemic carbinols $\mathbf{2 a - \mathbf { g }}$ in moderate-to-good yields. In order to investigate the hitherto only poorly investigated influence of esterification of antifungal hydroxyalkyl-imidazoles [11] and to introduce a chlorophenyl ring (cf. clotrimazole, Fig. 1) into the molecule, selected carbinols (2b and $\mathbf{2 c}$ ) were esterified with 4-chlorobenzoyl chloride [14] to give the esters $\mathbf{3 b}$ and $\mathbf{3 c}$. We also intended to achieve etherification of $\mathbf{2 a - g}$ with 2,4-dichlorobenzyl chloride (cf. miconazole, Fig. 1) by the use of a standard protocol with the bases sodium hydride or sodium ethoxide, but unfortunately did not observe any conversion.

\section{Biological Activity}

The antifungal and antibacterial activities of the resulting compounds were determined in an agar diffusion assay against four strains of bacteria (Gram-negative and Gram-positive) and four strains of fungi (two yeasts, mould, dermatophyte) [15]. Except for 2c, the compounds showed no or only weak activities against the bacteria, when compared to tetracycline, but especially compounds $\mathbf{2 c}, \mathbf{2 d}$, and $\mathbf{2 e}$, which contain $\mathrm{C}_{10}$ to $\mathrm{C}_{12}$-alkyl chains, show remarkable activity against fungi when compared to clotrimazole. The corresponding esters $\mathbf{3 b} / \mathbf{3 c}$ did not show significant antimicrobial activities. The MIC (minimum inhibitory concentration) against the opportunistic pathogen Candida glabrata, which is often associated with resistance to fluconazole therapy $[16,17]$, was determined to be $25 \mu \mathrm{g} / \mathrm{mL}$ (2c), $10 \mu \mathrm{g} / \mathrm{mL}$ (2d), and $5 \mu \mathrm{g} / \mathrm{mL}$ (2e) (clotrimazole: $2.5 \mu \mathrm{g} / \mathrm{mL}$ ) [11]. 
<smiles>Cn1cncc1C=O</smiles>

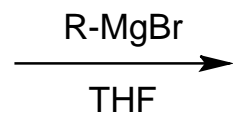

1<smiles>[R]C(O)c1cncn1C</smiles>

2a-g

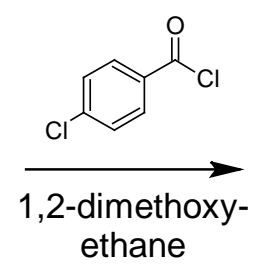<smiles>[R]C(OC(=O)c1ccc(Cl)cc1)c1cncn1C</smiles>

3b,c
a: $\mathrm{R}=\mathrm{C}_{5} \mathrm{H}_{11}$
b: $\mathrm{R}=\mathrm{C}_{6} \mathrm{H}_{13}$
c: $\mathrm{R}=\mathrm{C}_{9} \mathrm{H}_{19}$
d: $\mathrm{R}=\mathrm{C}_{10} \mathrm{H}_{21}$
e: $\mathrm{R}=\mathrm{C}_{11} \mathrm{H}_{23}$
f: $\mathrm{R}=\mathrm{C}_{12} \mathrm{H}_{25}$
a: $\mathrm{R}=\mathrm{CH}_{2} \mathrm{CH}_{2} \mathrm{Ph}$

Sch. 1. Synthetic preparation of the title compounds

To determinate the mechanism of action, the carbinols $\mathbf{2 c}, \mathbf{2} \mathbf{d}$, and $\mathbf{2 e}$ were evaluated in an ergosterol biosynthesis assay [18]. Only 2c showed a moderate accumulation of episterol (ergosta-7,24(28)-dien-3 $\beta$-ol) in this assay. This indicates that $\mathbf{2 c}$ is a moderate inhibitor of C5 desaturase, a hitherto poorly characterized, putatively non-heme ironcontaining oxidase [19]. But since the even more potent analogues $\mathbf{2 d}$ and $\mathbf{2 e}$ do not show signs of accumulation of episterol, the high antimycotic activity of the imidazolyl carbinols must be due to another mechanism of action.

Tab. 1. Agar diffusion assay (Te: tetracycline, $\mathrm{Cl}$ : clotrimazole, $50 \mu \mathrm{g} / \mathrm{disc}$, inhibition diameter in [mm], 0: no inhibition)

\begin{tabular}{lccccccccccc}
\hline & $\mathbf{2 a}$ & $\mathbf{2 b}$ & $\mathbf{2 c}$ & $\mathbf{2 d}$ & $\mathbf{2 e}$ & $\mathbf{2 f}$ & $\mathbf{2 g}$ & $\mathbf{3 b}$ & $\mathbf{3 c}$ & $\mathbf{T e}$ & $\mathbf{C l}$ \\
\hline Escherichia coli & 0 & 0 & $\mathbf{0}$ & 0 & 0 & 0 & 0 & 0 & 0 & $\mathbf{3 0}$ & $\mathbf{0}$ \\
Pseudomonas marginalis & 9 & 0 & $\mathbf{1 5}$ & 0 & 0 & 0 & 0 & 0 & 7 & $\mathbf{2 8}$ & $\mathbf{1 5}$ \\
Staphylococcus equorum & 15 & 0 & $\mathbf{2 0}$ & $\mathbf{1 8}$ & $\mathbf{1 3}$ & 10 & 0 & 7 & 0 & $\mathbf{3 7}$ & $\mathbf{2 0}$ \\
Streptococcus entericus & 0 & 0 & 7 & $\mathbf{1 0}$ & 8 & 7 & 0 & 7 & 0 & $\mathbf{2 0}$ & $\mathbf{8}$ \\
Candida glabrata & 0 & 0 & $\mathbf{1 0}$ & $\mathbf{1 5}$ & 9 & 7 & 0 & $\mathbf{1 0}$ & 6 & 0 & $\mathbf{1 9}$ \\
Aspergillus niger & 0 & 0 & 7 & $\mathbf{1 1}$ & 0 & 0 & 0 & $\mathbf{0}$ & 0 & 0 & $\mathbf{1 5}$ \\
Yarrowia lipolytica & 0 & 0 & $\mathbf{1 3}$ & $\mathbf{1 1}$ & 0 & 0 & 0 & 0 & 0 & 0 & $\mathbf{2 2}$ \\
Hyphopichia burtonii & 0 & 0 & $\mathbf{1 5}$ & $\mathbf{1 6}$ & $\mathbf{1 0}$ & 6 & 0 & $\mathbf{1 2}$ & 7 & 0 & $\mathbf{2 3}$ \\
\hline
\end{tabular}

\section{Conclusion}

The novel alkyl imidazolyl carbinols $\mathbf{2 c}, \mathbf{2 d}$, and $\mathbf{2 e}$ produced in this study show interesting antifungal activities. The investigation of a series of homologs indicated that the length of the alkyl chain is of critical importance, with an optimum length of $C_{10}$ to $C_{12}$. This parallels findings on $\mathrm{N}$-alkylimidazoles, where the same chain length was found to lead to the highest antibacterial and antifungal activity [20], and also on antifungal $N$-alkylmorpholines and -piperidines [9]. 
Although the main mechanism of action does not seem to be an inhibition of the ergosterol biosynthesis pathway (as initially intended), the pronounced antifungal activity of the compounds $\mathbf{2 c}, \mathbf{2 d}$, and $\mathbf{2 e}$ warrants them to be the starting point for the development of new antimycotics. The rather simple chemical structures of the novel compounds presented here is particularly interesting as they enable a quick, economical, and effortless synthesis approach to further analogues.

\section{Experimental}

\section{General}

Elemental analyses: Heraeus CHN-Rapid; IR-spectra: Perkin-Elmer FT-IR Paragon 1000; MS: Hewlett Packard MS-Engine; electron ionisation (El) $70 \mathrm{eV}$, chemical ionisation (CI) with $\mathrm{CH}_{4}(300 \mathrm{eV})$; NMR: Jeol GSX $400\left({ }^{1} \mathrm{H}: 400 \mathrm{MHz},{ }^{13} \mathrm{C}: 100 \mathrm{MHz}\right)$; melting points: Büchi Melting Point B-540 (not corrected); flash column chromatography (FCC): silica gel 60 (230-400 mesh, E. Merck, Darmstadt); GLC-MS: Shimadzu GC-17 A (carrier: He, oven temperature program: $100-280^{\circ} \mathrm{C}, 10^{\circ} \mathrm{C} / \mathrm{min}$, capillary column: Varian VF-5ms $30 \mathrm{~m} \times$ 0.25 , split injector $\mathrm{T}=250^{\circ} \mathrm{C}$, detector $\mathrm{T}=260^{\circ} \mathrm{C}$ ).

\section{General Procedure 1 (Grignard Reaction)}

$550 \mathrm{mg}(5.0 \mathrm{mmol})$ of 1-methyl-1H-imidazole-5-carbaldehyde (1) were dissolved in $20 \mathrm{~mL}$ dry THF and $7.5 \mathrm{mmol}$ of the Grignard reagent (solution in $50 \mathrm{~mL}$ anhydrous THF, freshly prepared from $7.5 \mathrm{mmol}$ of the corresponding alkyl or arylalkyl bromide, two crystals of iodine and $230 \mathrm{mg}$ (10 mmol) magnesium) were added dropwise. The mixture was stirred for $12 \mathrm{~h}$ at room temperature, then quenched with $30 \mathrm{~mL}$ of aqueous ammonia buffer $\mathrm{pH}$ 9.25 , and extracted with ethyl acetate $(3 \times 30 \mathrm{~mL})$. The combined organic layers were dried over $\mathrm{Na}_{2} \mathrm{SO}_{4}$ and the solvent was evaporated. The residue was purified by flash column chromatography ( $n$-hexane/ethyl acetate/dimethyl ethylamine 1:1:0.005).

\section{General Procedure 2 (Esterification)}

About $1.0 \mathrm{mmol}$ of carbinol $\mathbf{2 b / 2 c}$ was dissolved in $25 \mathrm{~mL}$ 1,2-dimethoxyethane and 4-chlorobenzoyl chloride (see amounts below) and $3 \mathrm{~mL}$ triethylamine (or pyridine) were added. The mixture was stirred for $12 \mathrm{~h}$ at room temperature. Then the solvent was evaporated, the residue dissolved in $25 \mathrm{~mL} 10 \%$ aqueous $\mathrm{NaOH}$ solution, and extracted with ethyl acetate $(3 \times 30 \mathrm{~mL})$. The combined organic layers were dried over $\mathrm{Na}_{2} \mathrm{SO}_{4}$ and the solvent was evaporated. The residue was purified by flash column chromatography ( $\mathrm{n}$ hexane/ethyl acetate 1:1).

\section{1-(1-Methyl-1H-imidazol-5-yl)hexan-1-ol (2a)}

The compound was prepared according "General Procedure 1" from $550 \mathrm{mg}$ (5 mmol) 1-methyl-1H-imidazole-5-carbaldehyde (1) and the Grignard reagent prepared from $1.13 \mathrm{~g}$ (7.5 mmol) 1-bromopentane to give $850 \mathrm{mg}(93 \%)$ of $2 \mathrm{a}$ as a white solid. M.p. $65^{\circ} \mathrm{C}$. ${ }^{1} \mathrm{H}-\mathrm{NMR}\left(\mathrm{d}_{6}\right.$-acetone): $\delta 0.88\left(\mathrm{t}, \mathrm{J}=7.3 \mathrm{~Hz}, 3 \mathrm{H}, \mathrm{CH}_{3}\right), 1.32\left(\mathrm{~m}, 6 \mathrm{H}, 3 \mathrm{CH}_{2}\right), 1.83(\mathrm{~m}, 2 \mathrm{H}$, $\left.\mathrm{CH}_{2}\right), 3.70\left(\mathrm{~s}, 3 \mathrm{H}, \mathrm{CH}_{3}\right), 4.62(\mathrm{t}, \mathrm{J}=6.7 \mathrm{~Hz}, 1 \mathrm{H}, \mathrm{CH}), 6.75(\mathrm{~s}, 1 \mathrm{H}$, aromat. $\mathrm{CH}), 7.36(\mathrm{~s}, 1$ $\mathrm{H}$, aromat. $\mathrm{CH}) .{ }^{13} \mathrm{C}$-NMR (d $\mathrm{d}_{6}$-acetone): $\delta 14.3\left(\mathrm{CH}_{3}\right), 23.3\left(\mathrm{CH}_{2}\right), 26.6\left(\mathrm{CH}_{2}\right), 31.9\left(\mathrm{CH}_{2}\right)$ $31.9\left(\mathrm{CH}_{3}\right), 32.4\left(\mathrm{CH}_{2}\right), 65.1(\mathrm{CH}), 126.6$ (aromat. $\left.\mathrm{CH}\right), 135.6$ (quart. C), 139.2 (aromat. $\mathrm{CH})$. MS (Cl) (m/z, \%): $183\left(\mathrm{M}^{+}+1,90\right), 165$ (100). MS (El) (m/z, \%): $182\left(\mathrm{M}^{+}, 10\right), 111$ 
(100). HR-MS Calcd. for $\mathrm{C}_{10} \mathrm{H}_{18} \mathrm{~N}_{2} \mathrm{O}:$ 182.1419. Found: 182.1428. IR (KBr), v, $\mathrm{cm}^{-1}: 3262$, 2951, 2854, 1508, 1416, 1237, 1072, 914.

\section{1-(1-Methyl-1H-imidazol-5-yl)heptan-1-ol (2b)}

The compound was prepared according "General Procedure 1" from $550 \mathrm{mg}$ (5 mmol) 1-methyl-1H-imidazole-5-carbaldehyde (1) and the Grignard reagent prepared from $1.24 \mathrm{~g}$ (7.5 mmol) 1-bromohexane to give $820 \mathrm{mg}(84 \%)$ of $2 \mathrm{a}$ as a pale brown solid. M.p. $92{ }^{\circ} \mathrm{C}$. ${ }^{1} \mathrm{H}$-NMR (d $\mathrm{d}_{4}$-methanol): $\delta 0.90\left(\mathrm{t}, \mathrm{J}=6.8 \mathrm{~Hz}, 3 \mathrm{H}, \mathrm{CH}_{3}\right), 1.32\left(\mathrm{~m}, 8 \mathrm{H}, 4 \mathrm{CH}_{2}\right), 1.86(\mathrm{~m}, 2 \mathrm{H}$, $\left.\mathrm{CH}_{2}\right), 3.72\left(\mathrm{~s}, 3 \mathrm{H}, \mathrm{CH}_{3}\right), 4.64(\mathrm{t}, \mathrm{J}=6.9 \mathrm{~Hz}, 1 \mathrm{H}, \mathrm{CH}), 6.86(\mathrm{~s}, 1 \mathrm{H}$, aromat. $\mathrm{CH}), 7.55(\mathrm{~s}, 1$ $\mathrm{H}$, aromat. $\mathrm{CH}) .{ }^{13} \mathrm{C}-\mathrm{NMR}\left(\mathrm{d}_{4}\right.$-methanol): $\delta 14.4\left(\mathrm{CH}_{3}\right), 23.7\left(\mathrm{CH}_{2}\right), 27.1\left(\mathrm{CH}_{2}\right), 30.2\left(\mathrm{CH}_{2}\right)$, $32.2\left(\mathrm{CH}_{3}\right), 33.0\left(\mathrm{CH}_{2}\right), 36.8\left(\mathrm{CH}_{2}\right), 65.5(\mathrm{CH}), 126.3$ (aromat. $\left.\mathrm{CH}\right), 136.1$ (quart. $\left.\mathrm{C}\right), 139.8$ (aromat. $\mathrm{CH})$. MS (Cl) (m/z, \%): $197\left(\mathrm{M}^{+}+1,100\right), 111$ (21). HR-MS Calcd.: 196.1576. Found: 196.1580. IR (KBr), v, cm ${ }^{-1}$ : 3107, 2952, 1508, 1467, 1413, 1233, 1113, 1072 , 1006, 930, 853, 825, 799, 701, 663.

\section{1-(1-Methyl-1H-imidazol-5-yl)decan-1-ol (2c)}

The compound was prepared according "General Procedure 1" from $550 \mathrm{mg}$ (5 mmol) 1-methyl-1H-imidazole-5-carbaldehyde and the Grignard reagent prepared from $1.55 \mathrm{~g}$ (7.5 mmol) 1-bromononane to give $0.840 \mathrm{~g}(71 \%)$ of $2 \mathrm{c}$ as a white solid. ${ }^{1} \mathrm{H}-\mathrm{NMR}\left(\mathrm{CDCl}_{3}\right)$ : $\delta 0.88\left(\mathrm{t}, \mathrm{J}=6.7 \mathrm{~Hz}, 3 \mathrm{H}, \mathrm{CH}_{3}\right), 1.27\left(\mathrm{~m}, 14 \mathrm{H}, 7 \mathrm{CH}_{2}\right), 1.86\left(\mathrm{~m}, 2 \mathrm{H}, \mathrm{CH}_{2}\right), 3.69(\mathrm{~s}, 3 \mathrm{H}$, $\left.\mathrm{CH}_{3}\right), 4.61(\mathrm{t}, \mathrm{J}=6.9 \mathrm{~Hz}, 1 \mathrm{H}, \mathrm{CH}), 6.80(\mathrm{~s}, 1 \mathrm{H}$, aromat. $\mathrm{CH}), 7.30(\mathrm{~s}, 1 \mathrm{H}$, aromat. $\mathrm{CH})$. ${ }^{13} \mathrm{C}-\mathrm{NMR}\left(\mathrm{CDCl}_{3}\right): \delta 14.2\left(\mathrm{CH}_{3}\right), 22.7\left(\mathrm{CH}_{2}\right), 26.2\left(\mathrm{CH}_{2}\right), 26.2\left(\mathrm{CH}_{2}\right), 29.4\left(\mathrm{CH}_{2}\right), 29.5$ $\left(\mathrm{CH}_{2}\right), 29.7\left(\mathrm{CH}_{2}\right), 32.0\left(\mathrm{CH}_{3}\right), 32.1\left(\mathrm{CH}_{2}\right), 35.9\left(\mathrm{CH}_{2}\right), 65.1(\mathrm{CH}), 126.3$ (aromat. $\left.\mathrm{CH}\right)$, 134.5 (quart. C), 138.7 (aromat. $\mathrm{CH}$ ). $\mathrm{MS}(\mathrm{Cl})(\mathrm{m} / \mathrm{z}, \%): 239\left(\mathrm{M}^{+}+1,100\right), 111$ (21). HR-MS Calcd. for $\mathrm{C}_{14} \mathrm{H}_{26} \mathrm{~N}_{2} \mathrm{O}$ : 238.2045. Found: 238.2045. IR ( $\left.\mathrm{KBr}\right), \mathrm{v}, \mathrm{cm}^{-1}$ : 3265, 2921, 2852, 1512, 1473, 1083, 920, 668.

\section{1-(1-Methyl-1H-imidazol-5-yl)undecan-1-ol (2d)}

The compound was prepared according "General Procedure 1" from $550 \mathrm{mg}$ (5 mmol) 1-methyl-1 $\mathrm{H}$-imidazole-5-carbaldehyde and the Grignard reagent prepared from $1.66 \mathrm{~g}$ (7.5 mmol) 1-bromodecane to give $658 \mathrm{mg} \mathrm{(52 \% )}$ of $\mathbf{2 d}$ as a white solid. M.p. $97{ }^{\circ} \mathrm{C} .{ }^{1} \mathrm{H}-$ $\operatorname{NMR}\left(\mathrm{CDCl}_{3}\right): \delta 0.88\left(\mathrm{t}, \mathrm{J}=7.0 \mathrm{~Hz}, 3 \mathrm{H}, \mathrm{CH}_{3}\right), 1.27\left(\mathrm{~m}, 16 \mathrm{H}, 8 \mathrm{CH}_{2}\right), 1.89\left(\mathrm{~m}, 2 \mathrm{H}, \mathrm{CH}_{2}\right)$, $3.70\left(\mathrm{~s}, 3 \mathrm{H}, \mathrm{CH}_{3}\right), 4.63(\mathrm{t}, \mathrm{J}=7.1 \mathrm{~Hz}, 1 \mathrm{H}, \mathrm{CH}), 6.90(\mathrm{~s}, 1 \mathrm{H}$, aromat. $\mathrm{CH}), 7.37(\mathrm{~s}, 1 \mathrm{H}$, aromat. $\mathrm{CH}) \cdot{ }^{13} \mathrm{C}$-NMR $\left(\mathrm{d}_{4}\right.$-methanol): $\delta 14.1\left(\mathrm{CH}_{3}\right), 22.7\left(\mathrm{CH}_{2}\right), 26.1\left(\mathrm{CH}_{2}\right), 29.3\left(\mathrm{CH}_{2}\right)$, $29.4\left(\mathrm{CH}_{2}\right), 29.5\left(\mathrm{CH}_{2}\right), 29.6\left(2 \mathrm{CH}_{2}\right), 31.9\left(\mathrm{CH}_{2}\right), 31.9\left(\mathrm{CH}_{3}\right), 35.7\left(\mathrm{CH}_{2}\right), 65.4(\mathrm{CH}), 126.5$ (aromat. $\mathrm{CH}$ ), 134.1 (quart. C), 138.9 (aromat. $\mathrm{CH}$ ). $\mathrm{MS}$ (EI) (m/z, \%): $252\left(\mathrm{M}^{+}, 17\right), 111$ (100). HR-MS Calcd. for $\mathrm{C}_{15} \mathrm{H}_{28} \mathrm{~N}_{2} \mathrm{O}$ : 252.2202. Found: 252.2199. IR (KBr), v, $\mathrm{cm}^{-1}$ : 3103, 2917, 2851, 1509, 1467, 1112, 1083, 823, 661.

\section{1-(1-Methyl-1H-imidazol-5-yl)dodecan-1-ol (2e)}

The compound was prepared according "General Procedure 1" from $550 \mathrm{mg}$ (5 mmol) 1-methyl-1H-imidazole-5-carbaldehyde and the Grignard reagent prepared from $1.76 \mathrm{~g}$ (7.5 mmol) 1-bromoundecane to give $927 \mathrm{mg} \mathrm{(70 \% )} \mathrm{of} 2 \mathrm{e}$ as a white solid. M.p. $98{ }^{\circ} \mathrm{C} .{ }^{1} \mathrm{H}-$ $\operatorname{NMR}\left(\mathrm{CDCl}_{3}\right): \delta 0.88\left(\mathrm{t}, \mathrm{J}=6.7 \mathrm{~Hz}, 3 \mathrm{H}, \mathrm{CH}_{3}\right), 1.35\left(\mathrm{~m}, 18 \mathrm{H}, 9 \mathrm{CH}_{2}\right), 1.89\left(\mathrm{~m}, 2 \mathrm{H}, \mathrm{CH}_{2}\right)$, $3.70\left(\mathrm{~s}, 3 \mathrm{H}, \mathrm{CH}_{3}\right), 4.63(\mathrm{t}, \mathrm{J}=7.0 \mathrm{~Hz}, 1 \mathrm{H}, \mathrm{CH}), 6.87(\mathrm{~s}, 1 \mathrm{H}$, aromat. $\mathrm{CH}), 7.36(\mathrm{~s}, 1 \mathrm{H}$, aromat. $\mathrm{CH}) \cdot{ }^{13} \mathrm{C}-\mathrm{NMR}\left(\mathrm{CDCl}_{3}\right): \delta 14.1\left(\mathrm{CH}_{3}\right), 22.7\left(\mathrm{CH}_{2}\right), 26.1\left(\mathrm{CH}_{2}\right), 29.3\left(\mathrm{CH}_{2}\right), 29.4$ $\left(\mathrm{CH}_{2}\right), 29.5\left(\mathrm{CH}_{2}\right), 29.6\left(3 \mathrm{CH}_{2}\right), 31.9\left(\mathrm{CH}_{2}\right), 31.9\left(\mathrm{CH}_{3}\right), 35.7\left(\mathrm{CH}_{2}\right), 65.2(\mathrm{CH}), 126.4$ 
(aromat. CH), 134.1 (quart. C), 138.8 (aromat. CH). MS (El) (m/z, \%): $248\left(\mathrm{M}^{+}-18,20\right)$, 219 (10), 135 (15), 121 (100), 108 (30). HR-MS Calcd. for $\mathrm{C}_{16} \mathrm{H}_{30} \mathrm{~N}_{2} \mathrm{O}$ : 266.2358. Found: 266.2347. IR (KBr), v, cm ${ }^{-1}$ : 3269, 2918, 2851, 1513, 1472, 1236, 1111, 1089, 1068, 904, 668.

\section{1-(1-Methyl-1H-imidazol-5-yl)tridecan-1-ol (2f)}

The compound was prepared according "General Procedure 1" from $550 \mathrm{mg}$ (5 mmol) 1-methyl-1H-imidazole-5-carbaldehyde and the Grignard reagent prepared from $1.87 \mathrm{~g}$ (7.5 mmol) 1-bromododecane to give $895 \mathrm{mg}(64 \%)$ of $2 \mathrm{f}$ as a white solid. M.p. $86{ }^{\circ} \mathrm{C} .{ }^{1} \mathrm{H}-$ NMR ( $\mathrm{d}_{4}$-methanol): $\delta 0.90\left(\mathrm{t}, \mathrm{J}=7.0 \mathrm{~Hz}, 3 \mathrm{H}, \mathrm{CH}_{3}\right), 1.32\left(\mathrm{~m}, 16 \mathrm{H}, 8 \mathrm{CH}_{2}\right), 1.86(\mathrm{~m}, 2 \mathrm{H}$, $\left.\mathrm{CH}_{2}\right), 3.72\left(\mathrm{~s}, 3 \mathrm{H}, \mathrm{CH}_{3}\right), 4.64(\mathrm{t}, \mathrm{J}=7.0 \mathrm{~Hz}, 1 \mathrm{H}, \mathrm{CH}), 6.86(\mathrm{~s}, 1 \mathrm{H}$, aromat. $\mathrm{CH}), 7.55(\mathrm{~s}, 1$ $\mathrm{H}$, aromat. $\mathrm{CH}) .{ }^{13} \mathrm{C}$-NMR $\left(\mathrm{d}_{4}\right.$-methanol): $\delta 14.4\left(\mathrm{CH}_{3}\right), 23.8\left(\mathrm{CH}_{2}\right), 27.2\left(\mathrm{CH}_{2}\right), 30.5\left(\mathrm{CH}_{2}\right)$, $30.6\left(\mathrm{CH}_{2}\right), 30.7\left(2 \mathrm{CH}_{2}\right), 30.8\left(3 \mathrm{CH}_{2}\right), 32.2(\mathrm{CH}), 33.1\left(\mathrm{CH}_{2}\right), 36.8\left(\mathrm{CH}_{2}\right), 65.5(\mathrm{CH})$, 126.3 (aromat. $\mathrm{CH}$ ), 136.1 (quart. C), 139.8 (aromat. $\mathrm{CH}) . \mathrm{MS}(\mathrm{Cl})(\mathrm{m} / \mathrm{z}, \%): 281\left(\mathrm{M}^{+}+1\right.$, 100), 263 (26). HR-MS Calcd. for $\mathrm{C}_{17} \mathrm{H}_{32} \mathrm{~N}_{2} \mathrm{O}$ : 280.2515. Found: 280.2516. IR ( $\left.\mathrm{KBr}\right), \mathrm{v}$, $\mathrm{cm}^{-1}$ : 3103, 2921, 2850, 1510, 1470, 1270, 1111, 1070, 941, 823, 717, 664.

\section{1-(1-Methyl-1H-imidazol-5-yl)-3-phenyl-propan-1-ol (2g)}

The compound was prepared according "General Procedure 1" from $550 \mathrm{mg}$ (5 mmol) 1-methyl- $\mathrm{H}$-imidazole-5-carbaldehyde and the Grignard reagent prepared from $1.39 \mathrm{~g}$ (7.5 mmol) 1-bromo-2-phenylethane to give $455 \mathrm{mg} \mathrm{(42 \% )}$ of $\mathbf{2 g}$ as a viscous oil. ${ }^{1} \mathrm{H}-\mathrm{NMR}$ $\left(\mathrm{CDCl}_{3}\right)$ : $\delta 2.21\left(\mathrm{~m}, 2 \mathrm{H}, \mathrm{CH}_{2}\right), 2.75\left(\mathrm{~m}, 1 \mathrm{H}, \mathrm{CH}_{2}\right), 2.86\left(\mathrm{~m}, 1 \mathrm{H}, \mathrm{CH}_{2}\right), 4.62(\mathrm{dd}, \mathrm{J}=5.8 \mathrm{~Hz}$, $\mathrm{J}=8.2 \mathrm{~Hz}, 1 \mathrm{H}, \mathrm{CH}), 6.90(\mathrm{~s}, 1 \mathrm{H}$, aromat. $\mathrm{CH}), 7.20(\mathrm{~m}, 3 \mathrm{H}, 3$ aromat. $\mathrm{CH}), 7.28(\mathrm{~m}, 2 \mathrm{H}$, 2 aromat. $\mathrm{CH}), 7.44(\mathrm{~s}, 1 \mathrm{H}$, aromat. $\mathrm{CH}) .{ }^{13} \mathrm{C}-\mathrm{NMR}\left(\mathrm{CDCl}_{3}\right): \delta 32.1\left(\mathrm{CH}_{3}\right), 32.2\left(\mathrm{CH}_{2}\right)$, $37.2\left(\mathrm{CH}_{2}\right), 64.1(\mathrm{CH}), 125.4$ (aromat. $\left.\mathrm{CH}\right), 126.1$ (aromat. $\left.\mathrm{CH}\right), 128.5$ (2 aromat. $\mathrm{CH}$ ), 128.5 (2 aromat. $\mathrm{CH}$ ), 134.2 (quart. C), 138.5 (aromat. $\mathrm{CH}$ ), 141.2 (quart. C). MS (EI) (m/z, \%): $216\left(\mathrm{M}^{+}, 10\right), 111$ (100), 83 (35). MS (Cl) (m/z, \%): $217\left(\mathrm{M}^{+}+1,100\right), 199$ (23). HR-MS Calcd. for $\mathrm{C}_{13} \mathrm{H}_{16} \mathrm{~N}_{2} \mathrm{O}: 216.1263$. Found: 216.1262.

\section{4-Chlorobenzoic acid 1-(1-methyl-1H-imidazol-5-yl)heptyl ester (3b)}

The compound was prepared according "General Procedure 2" from $232 \mathrm{mg}$ (1.18 mmol) $\mathbf{2 b}$ and $964 \mathrm{mg}$ (5.5 mmol) 4-chlorobenzoyl chloride to give $113 \mathrm{mg} \mathrm{(29 \% )} \mathrm{of} \mathbf{3 b}$ as a pale yellow oil. ${ }^{1} \mathrm{H}-\mathrm{NMR}\left(\mathrm{CDCl}_{3}\right)$ : $\delta 0.87\left(\mathrm{t}, \mathrm{J}=6.9 \mathrm{~Hz}, 3 \mathrm{H}, \mathrm{CH}_{3}\right), 1.31\left(\mathrm{~m}, 8 \mathrm{H}, 4 \mathrm{CH}_{2}\right), 1.90(\mathrm{~m}$, $\left.2 \mathrm{H}, \mathrm{CH}_{2}\right), 6.10(\mathrm{t}, \mathrm{J}=7.5 \mathrm{~Hz}, 1 \mathrm{H}, \mathrm{CH}), 7.14(\mathrm{~s}, 1 \mathrm{H}$, aromat. $\mathrm{CH}), 7.41(\mathrm{~s}, 1 \mathrm{H}$, aromat. $\mathrm{CH}), 7.41(\mathrm{~d}, \mathrm{~J}=8.6 \mathrm{~Hz}, 2 \mathrm{H}, 2$ aromat. $\mathrm{CH}), 7.95(\mathrm{~d}, \mathrm{~J}=8.6 \mathrm{~Hz}, 2 \mathrm{H}, 2$ aromat. $\mathrm{CH}) .{ }^{13} \mathrm{C}-$ NMR $\left(\mathrm{CDCl}_{3}\right)$ : $\delta 14.0\left(\mathrm{CH}_{3}\right), 22.5\left(\mathrm{CH}_{2}\right), 25.7\left(\mathrm{CH}_{2}\right), 28.9\left(\mathrm{CH}_{2}\right), 31.6\left(\mathrm{CH}_{2}\right), 32.0\left(\mathrm{CH}_{3}\right)$; $33.8\left(\mathrm{CH}_{2}\right), 67.3(\mathrm{CH}), 1283$ (quart. $\mathrm{C}$ ), 128.8 (aromat. $\left.\mathrm{CH}\right), 128.9$ (2 aromat. $\mathrm{CH}$ ); 130.6 (quart. C), 131.1 (2 aromat. CH); 138.9 (aromat. CH), 139.7 (quart. C). 165.2 (CO). MS (El) (m/z, \%): $335\left(\mathrm{M}^{+}+1,28\right), 239$ (31), 179 (100). HR-MS Calcd. for $\mathrm{C}_{18} \mathrm{H}_{23} \mathrm{ClN}_{2} \mathrm{O}_{2}$ : 334.1448. Found: 334.1450. IR (KBr), v, $\mathrm{cm}^{-1}$ : 2954, 2928, 2857, 1716, 1593, 1502, 1487, 1466, 1401, 1334, 1268, 1098, 1014, 760.

\section{4-Chlorobenzoic acid 1-(1-methyl-1H-imidazol-5-yl)decyl ester (3c)}

The compound was prepared according "General Procedure 2" from $235 \mathrm{mg}$ (0.99 mmol) 2c and $310 \mathrm{mg}$ (1.8 mmol) 4-chlorobenzoyl chloride to give $90 \mathrm{mg}(24 \%)$ of 3c as a almost colourless oil. ${ }^{1} \mathrm{H}-\mathrm{NMR}\left(\mathrm{CDCl}_{3}\right): \delta 0.84\left(\mathrm{t}, \mathrm{J}=7.2 \mathrm{~Hz}, 3 \mathrm{H}, \mathrm{CH}_{3}\right), 1.21\left(\mathrm{~m}, 14 \mathrm{H}, 7 \mathrm{CH}_{2}\right)$, $2.06\left(\mathrm{~m}, 2 \mathrm{H}, \mathrm{CH}_{2}\right), 3.65\left(\mathrm{~s}, 3 \mathrm{H}, \mathrm{CH}_{3}\right), 6.07(\mathrm{t}, \mathrm{J}=7.2 \mathrm{~Hz}, 1 \mathrm{H}, \mathrm{CH}), 7.12(\mathrm{~s}, 1 \mathrm{H}$, aromat. 
$\mathrm{CH}), 7.38(\mathrm{~d}, \mathrm{~J}=8.5 \mathrm{~Hz}, 2 \mathrm{H}, 2$ aromat. $\mathrm{CH}), 7.39(\mathrm{~s}, 1 \mathrm{H}$, aromat. $\mathrm{CH}), 7.92(\mathrm{~d}, \mathrm{~J}=8.5$ $\mathrm{Hz}, 2 \mathrm{H}, 2$ aromat. $\mathrm{CH}) .{ }^{13} \mathrm{C}-\mathrm{NMR}\left(\mathrm{CDCl}_{3}\right)$ : $\delta 14.0\left(\mathrm{CH}_{3}\right), 22.5\left(\mathrm{CH}_{2}\right), 25.7\left(\mathrm{CH}_{2}\right), 29.1$ (2 $\left.\mathrm{CH}_{2}\right), 29.3\left(2 \mathrm{CH}_{2}\right), 31.7\left(\mathrm{CH}_{2}\right), 31.9\left(\mathrm{CH}_{3}\right), 33.7\left(\mathrm{CH}_{2}\right), 67.2(\mathrm{CH}), 128.2$ (quart. C), 128.7 (2 aromat. $\mathrm{CH}$ ), 128.8 (aromat. $\mathrm{CH}$ ), 130.5 (quart. C), 130.9 (2 aromat. $\mathrm{CH}$ ), 138.8 (quart. C), 139.6 (aromat. CH), 165.0 (CO). MS (EI) (m/z, \%): $377\left(\mathrm{M}^{+}+1,70\right), 221$ (100). HR-MS Calcd. for $\mathrm{C}_{21} \mathrm{H}_{29} \mathrm{CIN}_{2} \mathrm{O}_{2}$ : 376.1918. Found: 376.1917. IR (KBr), v, $\mathrm{cm}^{-1}$ : 2926, 2854, 1716, 1594, 1496, 1458, 1398, 1275, 1171, 1095, 1017, 920, 852, 830, 767.

\section{Agar Diffusion Assay (DIN Method)}

The bacteria and fungi were cultivated on AC agar (Sigma). The substances were placed on $6 \mathrm{~mm}$ paper discs on the agar, each impregnated with $50 \mu \mathrm{g}$ of the tested compound or $50 \mu \mathrm{g}$ of the reference drugs. The bacteria media were incubated for $24 \mathrm{~h}$ at $32{ }^{\circ} \mathrm{C}$; the fungi media for $48 \mathrm{~h}$ at $28^{\circ} \mathrm{C}$, and the diameter of the zone of inhibition [mm] was then registered [15].

\section{Determination of MIC (DIN Method)}

$99 \mu \mathrm{L}$ of a suspension of Candida glabrata $\left(1 \times 10^{3} \mathrm{CFU} / \mathrm{ml}\right)$ in All Culture media (AC Agar, Aldrich) were incubated with $1 \mu \mathrm{L}$ ethanolic test solution for $36 \mathrm{~h}$ at $28{ }^{\circ} \mathrm{C}$ in a 96 well plate. After $36 \mathrm{~h}$, the turbidity was measured at $590 \mathrm{~nm}$ and compared to the cell suspensions without the substance and AC agar media [15].

\section{Acknowledgement}

The authors wish to thank Martina Stadler for her technical support with the bacterial cultures.

\section{Authors' Statement}

\section{Competing Interests}

The authors declare no conflict of interest.

\section{References}

[1] Benveniste P.

Sterol biosynthesis.

Ann Rev Plant Physiol. 1986; 37: 275-308.

http://dx.doi.org/10.1146/annurev.pp.37.060186.001423

[2] Bastert J, Schaller M, Korting HC, Evans EGV.

Current and future approaches to antimycotic treatment in the era of resistant fungi and immunocompromised hosts.

Int J Antimicrob Agents. 2001; 17: 81-91.

http://dx.doi.org/10.1016/S0924-8579(00)00323-X

[3] Taton M, Benveniste P, Rahier A.

Use of rationally designed inhibitors to study sterol and triterpenoid biosynthesis.

Pure Appl Chem 1987; 59: 287-294.

http://dx.doi.org/10.1351/pac198759030287 
[4] Song Z, Nes WD.

Sterol biosynthesis inhibitors: Potential for transition state analogs and mechanism-based inactivators targeted at sterol methyltransferase.

Lipids. 2007; 42: 15-33.

http://dx.doi.org/10.1007/s11745-006-3017-1

[5] Burden RS, Cooke DT, Carter GA.

Inhibitors of sterol biosynthesis and growth in plants and fungi.

Phytochemistry. 1989; 28: 1791-1804.

http://dx.doi.org/10.1016/S0031-9422(00)97862-2

[6] Renard D, Perruchon J, Giera M, Müller J, Bracher F.

Side chain azasteroids and thiasteroids as sterol methyltransferase inhibitors in ergosterol biosynthesis.

Biorg Med Chem. 2009; 17: 8123-8137.

http://dx.doi.org/10.1016/j.bmc.2009.09.037

[7] Van den Bossche H, Willemsens G, Cools W, Lauwers WFJ,Le Jeune L.

Biochemical effects of miconazole on fungi. II. Inhibition of ergosterol biosynthesis in Candida albicans.

Chem Biol Interact. 1978; 21: 59-78.

http://dx.doi.org/10.1016/0009-2797(78)90068-6

[8] Dolle RE, Allaudeen H S, Kruse LI.

Design and synthesis of 14- $\alpha$-methyl-15-aza-homosterols as novel antimycotics.

J Med Chem. 1990; 33: 817-880.

http://dx.doi.org/10.1021/jm00164a065

[9] Baloch RI, Mercer El, Wiggins TE, Baldwin BC.

Inhibition of ergosterol biosynthesis in Saccharomyces cerevisiae and Ustilago maydis by tridemorph, fenpropimorph and fenpropidin.

Phytochemistry. 1984; 23: 2219-2226.

http://dx.doi.org/10.1016/S0031-9422(00)80523-3

[10] Zirngibl L.

Antifungal azoles.

Weinheim: Wiley-VCH, 1994.

[11] Wahbi Y, Tournaire C, Cajoulle R, Payard M, Linas MD, Seguela JP.

Aliphatic ethers and esters of 1-(2,4-dichlorophenyl)-2-(1H-imidazolyl)ethanol: study of antifungal activity against yeasts and hydrophobic character.

Eur J Med Chem. 1994; 29: 701-706.

http://dx.doi.org/10.1016/0223-5234(94)90032-9

[12] Kelly SL, Lamb DC, Baldwin BC, Corran AJ, Kelly DE.

Characterization of Saccharomyces cerevisiae CYP61, sterol $\Delta^{22}$-desaturase, and inhibition by azole antifungal agents.

J Biol Chem. 1997; 272: 9986-9988.

http://dx.doi.org/10.1074/jbc.272.15.9986

[13] Ator MA, Schmidt SJ, Adams JL, Dolle RE, Kruse LI, Frey CL, Barone JM.

Synthesis, specifity, and antifungal activity of inhibitors of the Candida albicans $\Delta^{24}$-sterol

methyltransferase.

J Med Chem. 1992; 35: 100-106.

http://dx.doi.org/10.1021/jm00079a012

[14] Krauss J, Unterreitmeier D.

Synthesis of new lipophilic ipomeanol analogues and their cytotoxic activities.

Arch Pharm Pharm Med Chem. 2005; 338: 44-48.

http://dx.doi.org/10.1002/ardp.200400916 
[15] DIN Deutsches Institut für Normung e.V.

Methoden zur Empfindlichkeitsprüfung von mikrobiellen Krankheitserregern gegen

Chemotherapeutika.

DIN 58940, Teil 3 - Beiblatt 1 und Teil 4 - Beiblatt 1, Beuth Verlag, Berlin 1998.

[16] Bodey GP, Mardani M, Hanna HA, Boktour M, Abbas J, Girgawy E, Hachem RY, Kontoyiannis DP, Raad II.

The epidemiology of Candida glabrata and Candida albicans fungemia in immunocompromised patients with cancer.

Am J Med. 2002; 112: 380-385.

http://dx.doi.org/10.1016/S0002-9343(01)01130-5

[17] Lee I, Fishman NO, Zaoutis TE, Morales KH, Weiner MG, Synnestvedt M, Nachamkin I, Lautenbach $\mathrm{E}$.

Risk factors for fluconazole-resistant Candida glabrata bloodstream infections.

Arch Intern Med. 2009; 169: 379-383.

http://dx.doi.org/10.1001/archinte.169.4.379

[18] Müller C, Staudacher V, Krauss J, Giera M, Bracher F.

A convenient cellular assay for the identification of the molecular target of ergosterol biosynthesis inhibitors and quantification of their effects on total cholesterol biosynthesis.

Steroids. 2013; 78: 483-493.

http://dx.doi.org/10.1016/j.steroids.2013.006

[19] Goldstein AS.

Synthesis and bioevaluation of $\Delta^{7}$-5-desaturase inhibitors, an enzyme late in the biosynthesis of fungal sterol ergosterol.

J Med Chem. 1996; 39: 5092-5099.

http://dx.doi.org/10.1021/jm9605851

[20] de Savignac A, Roques C, Hinedi M, Michel G, Lattes A.

Synthése et propriétés antibactériennes et antifongiques d'une série de 1-alkylimidazoles.

Eur J Med Chem. 1990; 25: 449-454.

http://dx.doi.org/10.1016/0223-5234(90)90009-R 